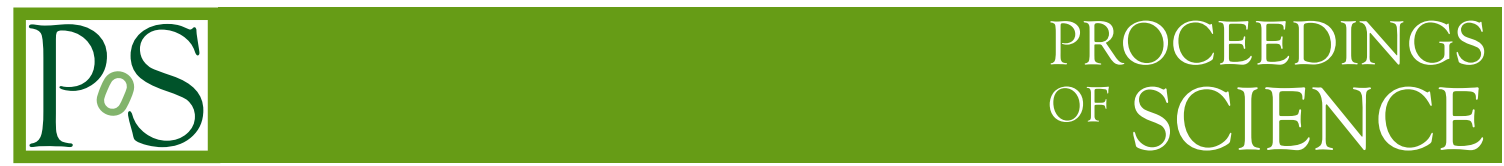

\title{
Triviality of $\phi_{4}^{4}$ in the broken phase revisited
}

\section{Tomasz Korzec and Ulli Wolff*}

Humboldt Universitat zu Berlin, Institut fur Physik, Newtonstrasse 15, D-12489 Berlin, Germany

E-mail: korzec@physik.hu-berlin.de, uwolffephysik.hu-berlin.de

We define a finite size renormalization scheme for $\phi^{4}$ theory which in the thermodynamic limit reduces to the standard scheme used in the broken phase. We use it to re-investigate the question of triviality for the four dimensional infinite bare coupling (Ising) limit. The relevant observables all rely on two-point functions and are very suitable for a precise estimation with the worm algorithm. This contribution updates an earlier publication by analysing a much larger dataset.

The 32nd International Symposium on Lattice Field Theory 23-28 June, 2014

Columbia University New York, NY

${ }^{*}$ Speaker. 


\section{Summary of theoretical background}

This brief article gives an update on [1] in the sense that we here analyze a very much enlarged new data set. This is found in the next section while we here summarize the theory for the reader's convenience ${ }^{1}$.

We consider the single component $\mathrm{Z}(2)$ symmetric scalar field theory on a torus of length $L$ embedded in four dimensional Euclidean space. We employ the simplest hypercubic lattice discretization with $L / a$ sites in each direction and the standard nearest neighbor lattice action

$$
S=\sum_{x}\left[\varphi(x)^{2}+\lambda\left(\varphi(x)^{2}-1\right)^{2}\right]-2 \kappa \sum_{x \mu} \varphi(x) \varphi(x+\hat{\mu}) .
$$

The standard picture for this quantum field theory is $[3,4]$ that there is a critical line $\kappa_{c}(\lambda)$ where the model possesses a continuum limit. This limit in $\kappa$ may be approached from below to reach the symmetric massive continuum theory or (for $\lambda>0$ ) from above to define the brokensymmetry massive model. The latter is of particular theorectical interest here due to some similarity to the Higgs field in the standard model.

As a standard way to renormalize the infinite volume theory in the broken phase we may match the Fourier transform $G(p)$ of the two point correlation function

$$
G(p)=\sum_{x} \mathrm{e}^{-i p x}\langle\varphi(x) \varphi(0)\rangle
$$

to the asymptotic form

$$
G(p)=Z\left\{v^{2} \delta^{4}(p)+\frac{1}{\hat{p}^{2}+m^{2}+\mathrm{O}\left(\hat{p}^{4}\right)}\right\} \quad(p \rightarrow 0)
$$

in the limit of vanishing $p$. Here the lattice momenta implied by our discretization

$$
\hat{p}_{\mu}=\frac{2}{a} \sin \left(a p_{\mu} / 2\right)
$$

have entered. In this formula $v$ and $m$ are the renormalized vacuum expectation value and the renormalized mass and $Z$ is a multiplicative renormalization factor. We prefer to define $v$ from a zero momentum contribution to the unsubtracted two point function rather than from the direct expectation value $\langle\varphi\rangle$ because we thus avoid subleties with an otherwise necessary symmetry breaking external field and we prepare the extension of the scheme to a finite size system.

On a finite torus - lattice or continuum - all momentum components get quantized to integer multiples of $2 \pi / L$. We now focus on three momenta with the smallest mutually differing values of $p^{2}$

$$
\begin{aligned}
p & =(0,0,0,0) \\
p_{*} & =(1,0,0,0) \frac{2 \pi}{L} \\
p_{* *} & =(1,1,0,0) \frac{2 \pi}{L},
\end{aligned}
$$

\footnotetext{
${ }^{1}$ A more detailed elementary introduction can be found in [2].
} 
for which we enforce (1.3) as exact equality and then solve for $Z, m, v$. The result is

$$
z^{2}=(m L)^{2}=\frac{G\left(p_{* *}\right) \hat{p}_{* *}^{2} L^{2}-G\left(p_{*}\right) \hat{p}_{*}^{2} L^{2}}{G\left(p_{*}\right)-G\left(p_{* *}\right)}
$$

and

$$
w^{2}=(v L)^{2}=\frac{G(0)}{G\left(p_{*}\right)} \frac{1}{L^{2} \hat{p}_{*}^{2}+z^{2}}-z^{-2} .
$$

where we have introduced the dimensionless finite size scaling quantities $z=m L$ and $w=v L$. In addition we have substitued

$$
\delta^{4}(p) \rightarrow L^{4} \delta_{p, 0}
$$

for the finite size system. A renormalized coupling in the broken phase is conveniently defined by the ratio of mass to expectation value,

$$
g=\frac{3 m^{2}}{v^{2}}=\frac{3 z^{2}}{w^{2}}
$$

In an expansion around one of the degenerate minima $g$ is seen to coincide with the bare coupling up to loop corrections.

In our numerical investigation we have restricted ourselves to the limit $\lambda \rightarrow \infty$. Then the path integral over lattice fields $\varphi$ with weight $\exp (-S)$ reduces to the Ising model where we sum over cofigurations $\{\varphi(x)= \pm 1\}$.

The required observable $G(p)$ can be very conveniently estimated in the loop representation [5] of the Ising model which is efficiently sampled by the worm algorithm [6]. In this ensemble the $\tanh (2 \kappa)$ expansion of

$$
\mathscr{Z}=\sum_{u, v} \sum_{\varphi} \mathrm{e}^{2 \kappa \sum_{x, \mu} \varphi(x) \varphi(x+\hat{\mu})} \varphi(u) \varphi(v)
$$

is sampled. As a consequence the distribution of $u$ and $v$ is related to the two point correlation,

$$
\langle\varphi(x) \varphi(0)\rangle=\frac{\left\langle\left\langle\delta_{x, u-v}\right\rangle\right\rangle}{\left\langle\left\langle\delta_{u, v}\right\rangle\right\rangle}
$$

where double angles refer to the average defined by (1.12). Finally the desired Fourier transforms are given by

$$
G(p)=\frac{\left\langle\left\langle\mathrm{e}^{-i p(u-v)}\right\rangle\right\rangle}{\left\langle\left\langle\delta_{u, v}\right\rangle\right\rangle}=\frac{\left\langle\left\langle\prod_{\mu} \cos \left(p_{\mu}(u-v)_{\mu}\right)\right\rangle\right\rangle}{\left\langle\left\langle\delta_{u, v}\right\rangle\right\rangle}
$$

where the reflection invariance in each direction is used to get a real representation in terms of cosines only.

\section{New data}

In comparison to [1] we have substantially extended our simulations. In table 1 we compile our complete dataset. Apart from some memory optimizations, our implementation of the worm algorithm is a standard one. The only 4D field that we keep in memory, is the link field that represents a graph of the $\tanh (2 \kappa)$ expansion. Since it can only assume two values per link, $V / 2$ bytes suffice for its storage. Thus even our largest lattices fit comfortably into the memory of a 


\begin{tabular}{rccll}
\hline$L / a$ & $2 \kappa$ & $z^{2}$ & \multicolumn{1}{c}{$g$} & $\left.\tilde{g}\right|_{z^{2}=10}$ \\
\hline 8 & 0.1524600 & $10.024(96)$ & $29.13(30)$ & $29.70(26)$ \\
12 & 0.1509920 & $10.008(98)$ & $24.88(26)$ & $25.09(22)$ \\
16 & 0.1504500 & $9.928(80)$ & $22.30(19)$ & $22.44(16)$ \\
24 & 0.1500460 & $9.974(98)$ & $19.65(21)$ & $19.70(18)$ \\
32 & 0.1498990 & $9.970(65)$ & $17.93(13)$ & $17.96(11)$ \\
48 & 0.1497900 & $10.189(53)$ & $16.121(93)$ & $16.074(77)$ \\
64 & 0.1497484 & $10.03(11)$ & $15.03(18)$ & $15.03(15)$ \\
80 & 0.1497294 & $10.10(14)$ & $14.36(22)$ & $14.34(19)$ \\
160 & 0.1497035 & $10.30(38)$ & $12.06(49)$ & $12.01(42)$ \\
\hline
\end{tabular}

Table 1: Simulation results for $z$ and $g$ at growing $L / a$ and for $\tilde{g}$ corrected to refer to $z^{2}=10$.

standard desktop PC. It took roughly $43 \mathrm{k}$ core hours to generate our most expensive $(L / a=160)$ ensemble. This corresponds to $1.2 \times 10^{7} \times V$ worm updates.

The coupling $\tilde{g}$ is related to the renormalized coupling $g$ by

$$
\tilde{g}=g \frac{1}{\left(1-a^{2} m^{2} / 16\right)^{2}},
$$

i. e. it only differs by a small lattice artefact. The rationale [1] is that the Callan Symanzik $\beta$ function for $g$ has a tree level artefact contribution which is absent for $\tilde{g}$. For our $z^{2}=10$ data this amounts to a relative $1.25 L^{-2}$ correction that is completely insignificant except for the smallest lattices.



Figure 1: Data and linear fit for $g^{-2} d g / d z$.

We had to tune $\kappa$ to approach $z^{2}=10$. Table 1 shows that we were often successful within our small statistical errors. To implement the remaining tiny correction leading to the last column we 
have numerically determined the derivative $d g / d\left(z^{2}\right)$. This is relatively easy by using the relation

$$
t \frac{d}{d t}\langle\langle A\rangle\rangle=\langle\langle A K\rangle\rangle-\langle\langle A\rangle\rangle\langle\langle K\rangle\rangle
$$

which holds in the loop representation [5]. In this formula $t \equiv \tanh (2 \kappa)$ is used, $A$ can be any $\kappa$-independent observable and $K$ is the total number of links occupied by lines. We see in figure 1 that beyond $L=64$ the connected correlation (2.2) is too noisy to get a signal and we had to extrapolate with the shown linear fit. Its form is suggested by perturbation theory. We emphasize however that any error in this procedure only affects a systematic correction in the final results that itself is only of the order of the statistical error.

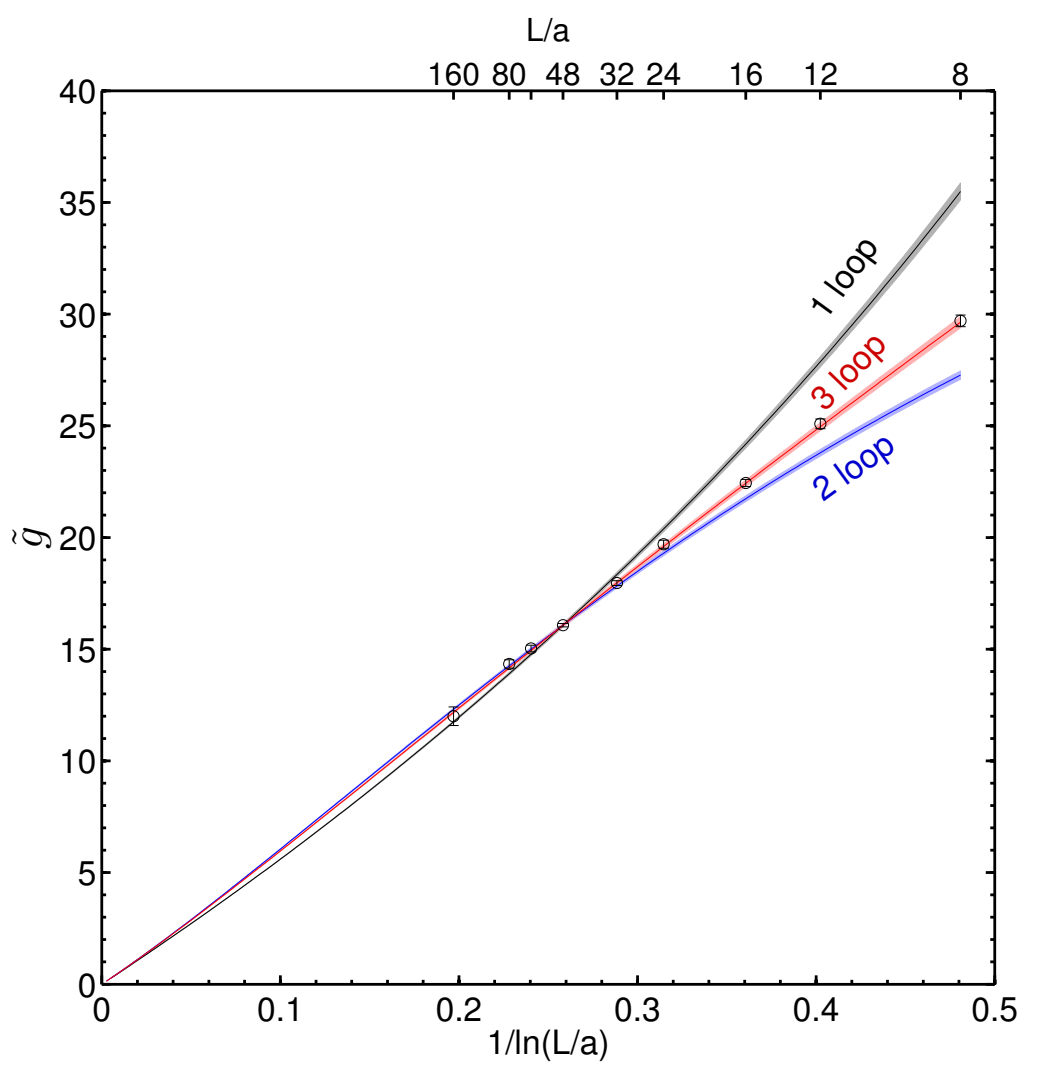

Figure 2: Cutoff dependence of the coupling $\tilde{g}$.

Our main result is now represented by figure 2 . The curves show the evolution with the perturbative renormalization group at 1,2 and 3 loop ${ }^{2}$ precision where the evolution is started (in both directions) from our most precise data point at $L / a=48$. The shaded bands represent the small error of the initial value. We see a perfect match of all our data points with the three loop evolution and thus complete consistency with the (logarithmic) triviality scenario.

\footnotetext{
${ }^{2}$ We have to note that the three loop term is taken from [4] and refers to $z=\infty$. According to experiences in the symmetric phase [7] the value for $z^{2}=10$ is expected to be very similar.
} 
Acknowledgements: We thank Peter Weisz for discussions and the Deutsche Forschungsgemeinschaft (DFG) for support in the framework of SFB Transregio 9.

\section{References}

[1] Johannes Siefert and Ulli Wolff, Triviality of $\phi_{4}^{4}$ theory in a finite volume scheme adapted to the broken phase, Phys. Lett. B 733 (2014) 11 [arXiv:1403.2570 [hep-lat]].

[2] Ulli Wolff Triviality of four dimensional $\phi^{4}$ theory on the lattice, (2014) Scholarpedia, 9(10):7367.

[3] M. Lüscher and P. Weisz, Scaling Laws and Triviality Bounds in the Lattice phi**4 Theory. 1. One Component Model in the Symmetric Phase, Nucl. Phys. B290 (1987) 25.

[4] M. Lüscher and P. Weisz, Scaling Laws and Triviality Bounds in the Lattice phi**4 Theory. 2. One Component Model in the Phase with Spontaneous Symmetry Breaking, Nucl.Phys. B295 (1988) 65.

[5] U. Wolff, Simulating the All-Order Strong Coupling Expansion I: Ising Model Demo, Nucl. Phys. B810 (2009) 491, [arXiv: 0808 . 3934].

[6] N. Prokof'ev and B. Svistunov, Worm Algorithms for Classical Statistical Models, Phys. Rev. Lett. 87 (2001) 160601, [arXiv:0910.1393].

[7] P. Weisz and U. Wolff, Triviality of $\phi_{4}^{4}$ theory: small volume expansion and new data, Nucl. Phys. B846 (2011) 316-337, [arXiv: 1012.0404 ]. 